\title{
Prospective blinded surveillance screening of Swedish women with increased hereditary risk of breast cancer
}

\author{
Annelie Liljegren ${ }^{1}$ (D) Anna von Wachenfeldt ${ }^{2} \cdot$ Edward Azavedo $^{3} \cdot$ Sandra Eloranta $^{4} \cdot$ Helene Grundström $^{5}$. \\ Anne Kinhult Ståhlbom ${ }^{1}$. Ann Sundbom ${ }^{6} \cdot$ Per Sundén $^{5}$. Gunilla Svane ${ }^{3}$. Dieter Ulitzsch ${ }^{5}$. Brita Arver ${ }^{1}$
}

Received: 3 October 2017 / Accepted: 23 December 2017 / Published online: 9 January 2018

(c) The Author(s) 2018. This article is an open access publication

\begin{abstract}
Purpose To evaluate the sensitivity and specificity of different screening modalities in women with a family history of breast cancer.

Methods Our blinded, prospective, comparative cohort analysis included three types of screening, mammography, ultrasound, and clinical breast examination once per year for 6 years. Eligible patients for this study were healthy women with $\geq 17 \%$ lifetime risk of breast cancer or with a mutation in BRCAl or BRCA2.

Results A total of 632 women were screened between 2002 and 2012 (each for 6 years). During the study, 30 women were diagnosed with breast cancer, with 10 of these diagnoses occurring between screening visits, and six of the 10 diagnosed women were gene carriers. The clinical presentation for the women diagnosed with breast cancer was followed until 2017. No consistent patterns for the diagnostic capacity of the different screening modalities were found, although mammography showed low sensitivity, whereas ultrasound showed better sensitivity in three of the six rounds. The specificity was high in mammography and improved in ultrasound over time. Most importantly, clinical breast examination provided no additional information toward the diagnosis of breast cancer.

Conclusion Neither mammography nor ultrasound performed yearly were sensitive enough as standalone modalities, although high specificity was confirmed. Our findings indicate that high risk (> 29\% life time risk) individuals and gene carriers can be screened biannually, using the same protocol as used in mutation carriers. Our results also suggest that low-risk groups $(<20 \%)$ may continue to be referred to population mammography screening program, while clinical breast examination may be omitted in all risk groups, and could be optional in gene carriers.
\end{abstract}

Keywords Hereditary breast cancer $\cdot$ Surveillance $\cdot$ Prevention program $\cdot$ Mammography $\cdot$ Ultrasound $\cdot$ BRCA1 $\cdot$ BRCA2

\section{Introduction}

Worldwide awareness of risk factors associated with a family history of breast cancer is increasing. Women at increased risk (often defined as doubled lifetime risk) are

Electronic supplementary material The online version of this article (https://doi.org/10.1007/s10549-017-4639-0) contains supplementary material, which is available to authorized users.

Annelie Liljegren

Annelie.liljegren@sll.se

1 Department of Oncology-Pathology, Karolinska University Hospital, Karolinska Institutet, Radiumhemmet, 17176 Stockholm, Sweden

2 Department of Oncology-Pathology, Karolinska University Hospital/Södersjukhuset, Stockholm, Sweden recommended to obtain regular surveillance after genetic counseling and risk assessment $[7,15]$. Women with mutations in the BRCA1, BRCA2, PALB2, or TP53 genes who are at considerably high risk for breast cancer are also informed about the possibility of risk-reducing surgery. Surveillance includes mammography, which may be used in combination with breast ultrasound, breast magnetic resonance imaging, and clinical breast examination. Breast self-examination is

3 Department of Radiology, Karolinska University Hospital, Karolinska Institutet, Stockholm, Sweden

4 Scandinavian Development Services, Danderyd, Sweden

5 Department of Radiology, Danderyd Hospital, Stockholm, Sweden

6 Department of Radiology, Södersjukhuset, Stockholm, Sweden 
also advocated. Limited sensitivity in detecting breast cancer with the use of only mammography $(32-41 \%)[10-13,20$, $21]$ or only ultrasound $(32-60 \%)[16,19]$ has been reported. Mammography used in conjunction with ultrasound has a higher, sensitivity ranging of $49 \%$ [11] to $77.5 \%$ [5] than mammography or ultrasound alone. The sensitivity of magnetic resonance imaging is superior to that of the other imaging methods [10-13] especially in young women and in BRCA1- or BRCA2-mutation carriers [20]. International guidelines advocate annual mammography to women with moderate (17-29\%) life time risk and annual mammography and magnetic resonance imaging to $B R C A 1$ - or $B R C A 2$ mutation carriers [15]. However, there has been no blinded study following a large cohort of women over multiple years to assess the importance of these test modalities in combination. The majority of the published surveillance studies report a follow-up of 1-4 years [15] and there is a lack of reports of long time surveillance in a cohort of women at increased risk due to hereditary breast cancer.

Therefore, we designed this blinded, prospective study with the aim to evaluate the sensitivity and specificity, i.e., the diagnostic capacity/effectiveness of the different screening tests upon imaging, of the different screening modalities (mammography, ultrasound, clinical breast examination) in women with a family history of breast cancer. The study compared screenings for 632 women using three types of screening, mammography, ultrasound, and clinical breast examination once per year for 6 years and ran from 2001 to 2012. Eligible patients for this study were healthy women with $\geq 17 \%$ lifetime risk of breast cancer or with a mutation in BRCA1 or BRCA2.

\section{Materials and methods}

\section{Study design and study center}

This prospective, comparative cohort study aimed to compare the diagnostic capacity of screening modalities among women with an increased risk of breast cancer due to family history. The surveillance included three types of screening, mammography, ultrasound, and clinical breast examination once per year for 6 years for each participant. Enrollment of the women in the study took place between 2002 and 2006 with the final screening visit for the last included patient in 2012. For patients that were diagnosed with breast cancer within the study, follow-up data regarding recurrences and death were collected from medical records up to October 2017 in order to give a descriptive view of survival so far. As only one magnetic resonance imaging unit in a developing phase was present in our region when this study was conducted, magnetic resonance imaging screening was not included in our study although since 2006, magnetic resonance imaging has been used for $B R C A 1 / 2$ mutation carriers and in individuals with a medical history of breast cancer according to international guidelines. Only descriptive data about the magnetic resonance imaging findings are presented in this report. Study participants were recruited from the Familial Cancer Centre, Oncology Department, Karolinska University Hospital. This centre has three site locations in Stockholm: Danderyds Hospital (Site 1), Karolinska University Hospital (Site 2), and Södersjukhuset (Site 3). Written informed consent was obtained from all participants, and the Ethics Committee at Karolinska Institutet approved the study on 15-10-2001 (no. 01-065).

\section{Patients}

Inclusion criteria: healthy women between the ages of 25 and 60 underwent genetic counseling and risk assessment. Women with $\geq 17 \%$ lifetime risk of breast cancer according to Claus tables [6], or women with a family history indicating an autosomal dominant disease of breast cancer, were eligible for the study. Those with a personal history of breast or ovarian cancer and a 5-year disease-free interval in combination with a family history for breast cancer were also included. In addition, healthy women with a mutation in BRCA1, BRCA2, PTEN, or TP53 were eligible. A normal mammogram 1 year before the first screening round was mandatory.

Exclusion criteria: Women with no known mutation in the family were not included if they were $>10$ years younger than the earliest age in which a family member was affected with breast cancer. Mutation carriers were not eligible if younger than 25 years of age.

A total of 656 women with a normal mammogram were initially included in the study. Twenty-four women declined participation before entering screening rounds.

The final number of women enrolled who started screening round 1 was 632 with a mean age of 44.1 (range 25-60). Over the course of 6 years, 95 women discontinued the study due to prophylactic mastectomy $(n=46), \operatorname{logistics}(n=13)$, migration $(n=12)$, tested as a non-carrier $(n=7)$, noncancer-related death $(n=3)$, and lost contact $(n=12)$. Of the 3792 planned screening visits, a total of 3478 screenings were performed (92\%).

\section{Genetic investigation}

The counseling procedure included a pedigree of family history with medical records confirming cancer diagnoses and death certificates or data from the Swedish Cancer Registry. Genetic screening of the BRCA1/2 genes was offered to families having one of the following characteristics according to the national guidelines: (1) at least three cases of breast or ovarian cancer in first- or second-degree relatives (with 
at least one relative younger than 50 at diagnosis); (2) two cases of breast or ovarian cancer in first- or second-degree relatives (at least one relative below age 40 at diagnosis); (3) one individual with breast or ovarian cancer diagnosed when less than 35 years of age; (4) close relatives with the combination of breast and ovarian cancer, regardless of the age of onset.

\section{Risk assessment}

In this study, the Claus risk tables [6] were used to determine lifetime risk of breast cancer in families with one or two close relatives with breast cancer. All pedigrees were grouped in relation to hereditary patterns (supplementary Table 1). The pedigrees were classified according to each study participant's life time risk of breast cancer, and four groups were defined: risk group 1-moderate risk of breast cancer; risk group 2-high risk of breast cancer; risk group 3-medical history of breast or ovarian cancer and at least one close relative with breast or ovarian cancer; risk group 4-very high risk, mutation carriers with or without a previous medical history of breast or ovarian cancer.

The risk estimation model Breast and Ovarian Analysis of Disease Incidence and Carrier Estimation Algorithm [BOADICEA] has replaced the Claus tables in clinical practice during the last decade. Thus, the lifetime risk for all women in the study cohort was calculated retrospectively according to BOADICEA version 2 [3] to investigate if that selection of participants by Claus tables was comparable to that by BOADICEA.

\section{Breast assessment}

The mammography, ultrasound, and clinical breast examination findings were scored on a five-point scale: $1=$ normal, $2=$ benign, $3=$ possibly malignant, $4=$ most probably malignant, and $5=$ malignant. This is a modified version of mammographic findings described by Azavedo et al. and frequently used in Sweden [4]. Mammographic density was estimated according to Wolfe's division of breast density into four groups of increasing density: N1, P1, P2, and DY [22]. When available, magnetic resonance images were categorized using the American College of Radiology Breast Imaging-Reporting and Data System (BI-RADS) categories and scored on a 5-point scale [1].

\section{Demographics and lifestyle assessment}

Self-administered questionnaires were collected at the start of the study to record demographic characteristics, history of gynecological and/or breast surgery, reproductive history, hormonal treatment, menopausal status, body mass index, smoking and alcohol habits, physical activity, and habits of self-examination.

\section{Procedure}

Mammography and ultrasound were performed no $<4$ weeks apart and before the clinical breast examination that was done within 4 weeks from the imaging. The most experienced ultrasound radiologists worked at Site 1 and all ultrasound procedures were performed here. Mammography was done either at Site 2 or 3. Clinical breast examination was performed at all three sites. To ensure the procedure was blinded, no communication among the radiologists and the clinicians involved in the study was allowed before the annual clinical breast examination. Additionally, no communication regarding findings from the screening was permitted between the radiologists and the study subject. After the clinical breast examination was performed, the results from the imaging modalities were disclosed and made available to all physicians involved in the diagnostic process. If there was an abnormality (i.e., code 3 or higher on any imaging examination modality), the study subject was referred for further diagnostics. Specifically, women with palpable lesions code 3 or more were referred for fine-needle biopsy. Referrals for cytology were optional for code 2 (code $2=$ benign) but was not included in the statistics on the sensitivity and specificity analyses. In pregnant or lactating women, only ultrasound imaging was used.

During the 6-year screening program, 24 women $(0.75 \%$ of all mammography imaging) were referred to further diagnostic investigation by the mammography breast radiologist, 213 (6.4\% of all ultrasound imaging) by the ultrasound breast radiologist, and 302 (9.1\% of all clinical breast examination) by the clinicians. Over time, there were fewer biopsy referrals from the clinicians and the ultrasound radiologists (Fig. 1).

\section{Screening modalities}

A total of five experienced breast radiologists examined mammography images. Each mammogram was examined by two different breast radiologists. Mammography was performed with two views per breast, Medio-lateral-oblique and craniocaudal using an analogue technique at both sites. At Site 2, an Instrumentarium Diamond machine was used. At Site 3, the Siemens Mammomat 300 was used until 2006 when it was replaced by Siemens Mammomat 3000 Nova in January 2007.

Ultrasound was performed using Linear Array $8.7-\mathrm{MHz}$ probes (L39, GE Logic 400). Since 2006, a high-resolution 5-13-MHz Linear Matrix Array probe (ML12, GE Logic 9) was increasingly used. Both breasts and the axillas were 


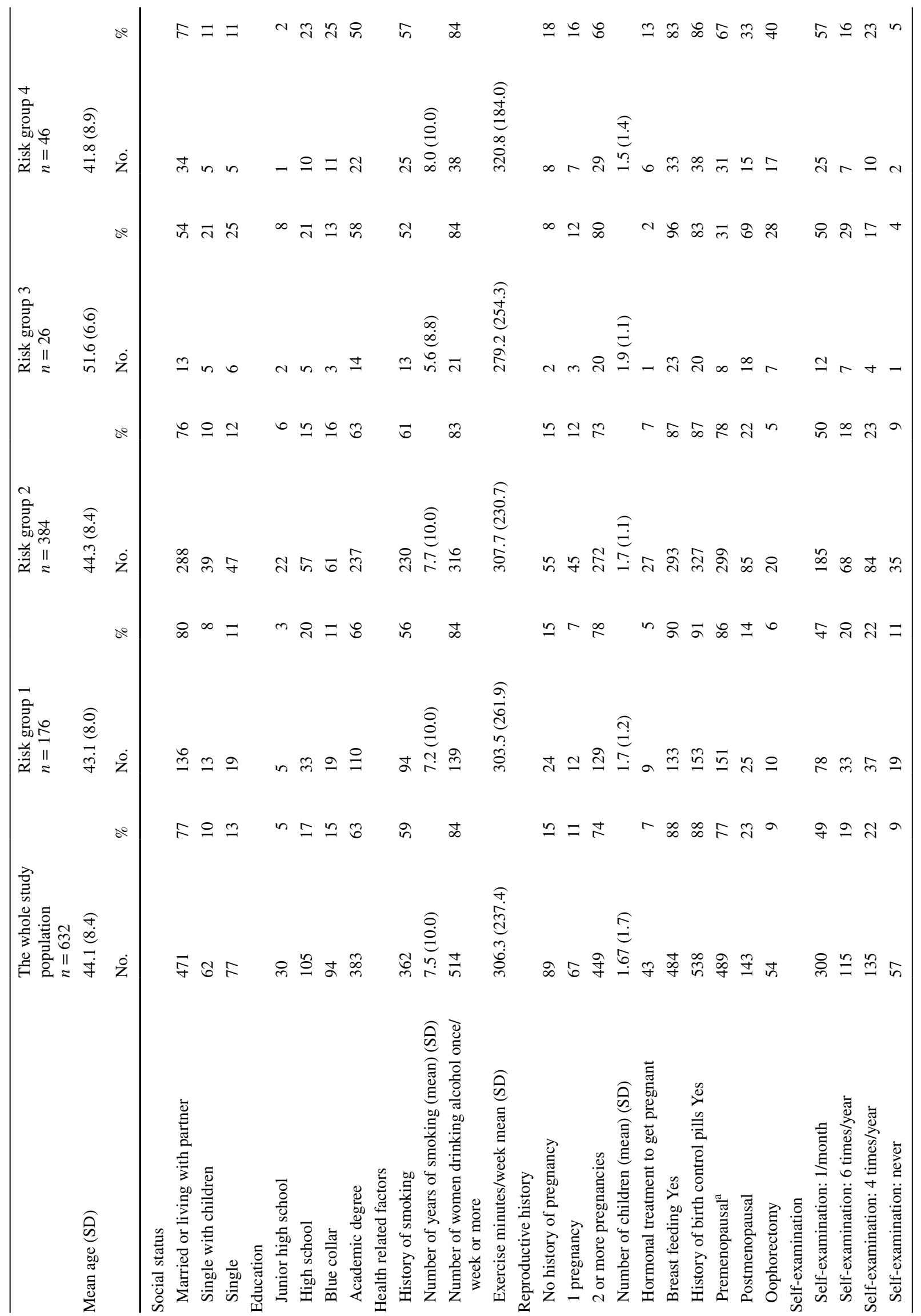




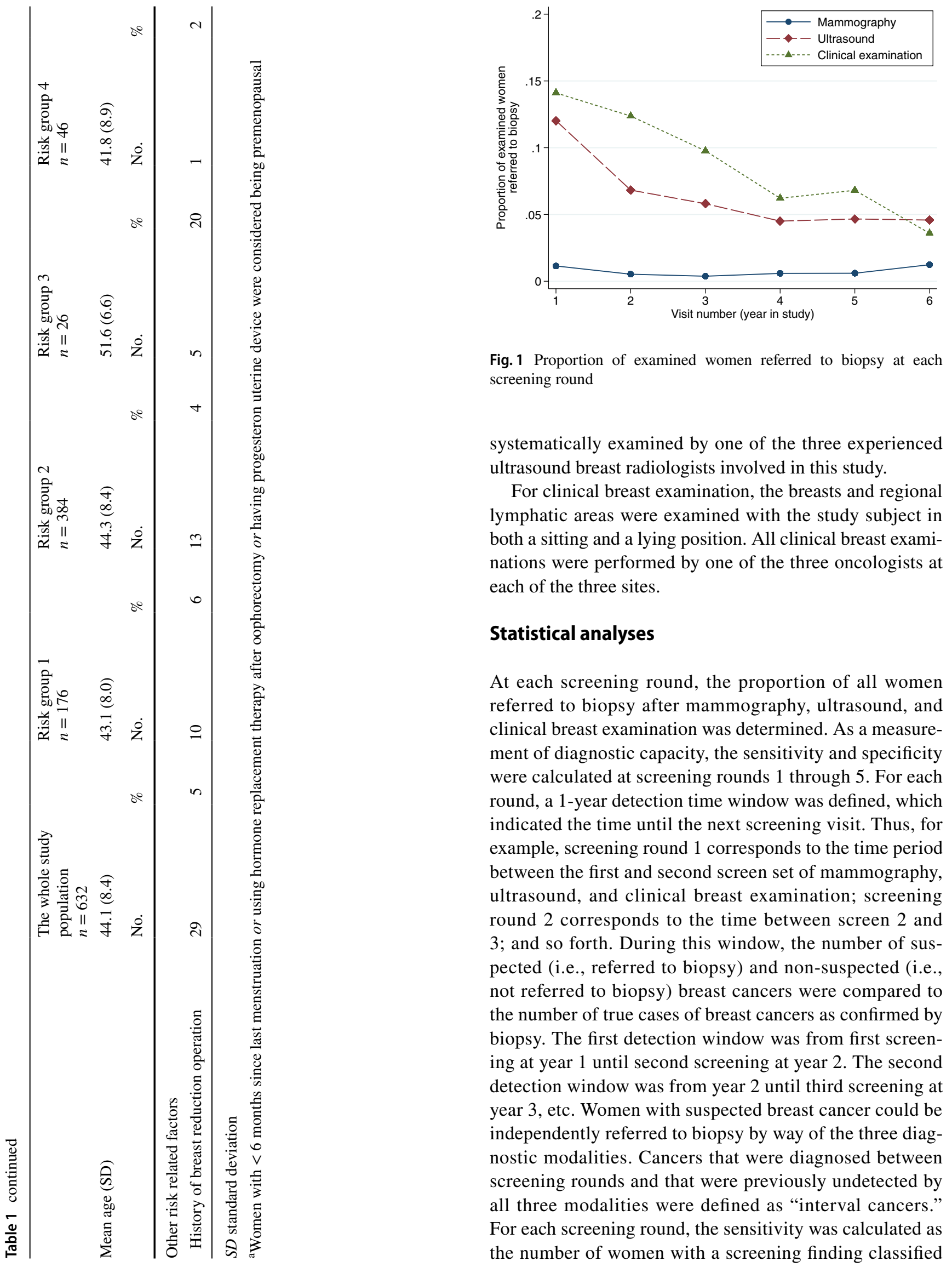


as code 3 or higher with a confirmed diagnosis of breast cancer divided by the total number of confirmed cases diagnosed within the detection window. The specificity was calculated as the number of breast cancer-free women who were not referred to biopsy at screening divided by the total number of women who were breast cancer-free (i.e., including false positives) during the same detection window.

The sensitivity and specificity at each screening round were used to produce non-parametric receiver operating characteristics curves for the three diagnostic modalities. The diagnostic capacity of each modality at each round was subsequently contrasted by calculating areas under the curve (AUC), including 95\% confidence intervals. All descriptive statistics were performed with the SPSS for Windows program, version 16.0. Statistical analyses of sensitivity and specificity were performed using the roctab and roccomp functions in Stata 13 [18].

\section{Results}

The baseline demographics of the women in the different risk groups and the results from the self-reported questionnaire administered at inclusion are presented in Table 1. The mean age was statistically significantly higher in risk group 3 (51.6) than in the other risk groups (41.8-43.1), $p<0.001$.

\section{Cancer detection}

In our study of at-risk women for breast cancer, thirty of the enrolled 632 women (4.7\%) were diagnosed with breast cancer at some time during the 6-year screening protocol. Ten of these tumors occurred between screening visits. Among these ten cancers, two were found incidentally upon the histological examination following prophylactic mastectomy, and two cases of breast cancer were found in two women, who by mistake participated in the concurrent mammography population screening program. Among the 10 women with interval breast cancers, three women were known gene carriers before entering the study while three were identified as carriers after breast cancer diagnose. Twenty-five of the 30 women diagnosed with breast cancer had invasive tumors. Of these, nine (36\%) were lymph node positive. Tumor characteristics, breast cancer recurrences, and years of survival up to October 2017 of the 30 patients diagnosed with breast cancer are presented in Table 2. Two patients died from breast cancer and two died from other cause during the follow-up time. One patient was diagnosed with contralateral breast cancer and one patient had a local recurrence. For further details see Table 2.

\section{Sensitivity, specificity, and area under the curve for the three screening modalities}

In screening round 1 (the detection window between year 1 and year 2), the diagnostic capacity of mammography and ultrasound was similar with an AUC for mammography of 0.57 (95\% CI $0.43-0.71)$ and for ultrasound of 0.58 (95\% CI 0.40-0.76). However, the AUC for clinical breast examination $(0.42,95 \%$ CI $0.42-0.44)$ was significantly lower than that for mammography and ultrasound $(p=0.0045)$.

In screening round 2 (the detection window between year 2 and year 3 ), there was no difference in diagnostic capacity between the three screening modalities.

In screening round 3, all three modalities resulted in AUCs of 0.50 or lower. Furthermore, the cancers that occurred during this time window were all interval cancers, i.e., undetected by all three screening modalities at the most recent visit. In screening round 4 , ultrasound had a higher AUC $(0.90,95 \%$ CI 0.74-1.00) compared to both mammography $(0.58,95 \% \mathrm{CI} 0.42-0.74, p=0.0026)$ and clinical breast examination $(0.64,95 \%$ CI $0.43-0.84, p=0.0189)$, respectively. This pattern was similar in screening round 5 , although the difference in AUCs for ultrasound (0.82, 95\% CI 0.49-1.00) and mammography (0.66, 95\% CI 0.34-0.99) was no longer statistically significant. The AUC for clinical breast examination $(0.47,95 \%$ CI $0.45-0.48)$ remained significantly lower than that of ultrasound $(p=0.0346)$ (Fig. 2).

\section{Risk assessment according to BOADICEA}

If the BOADICEA risk model had been used with the same cut off value of $\geq 17 \%$ as used in the present study 217 (34\%) of the women, of which five were diagnosed with breast cancer, would not have been eligible in this study. However, calculating the life time breast cancer risk for the five women diagnosed with breast cancer who did not reach $17 \%$ according to BOADICEA version 2, the BOADICEA version 3 captured all but one of these women with breast cancer.

\section{Discussion}

This is the first Swedish report of yearly surveillance in a large cohort of families with breast and/or ovarian cancer. In our cohort of 632 women, a total of 30 breast cancers were detected with 10 of these detected in between screening rounds. Five of these patients were undetected gene carriers when the study started. Among the three screening modalities, mammography showed a low sensitivity and high specificity. Ultrasound showed higher sensitivity in three out of five screening rounds. The specificity of ultrasound screening improved over subsequent screening 


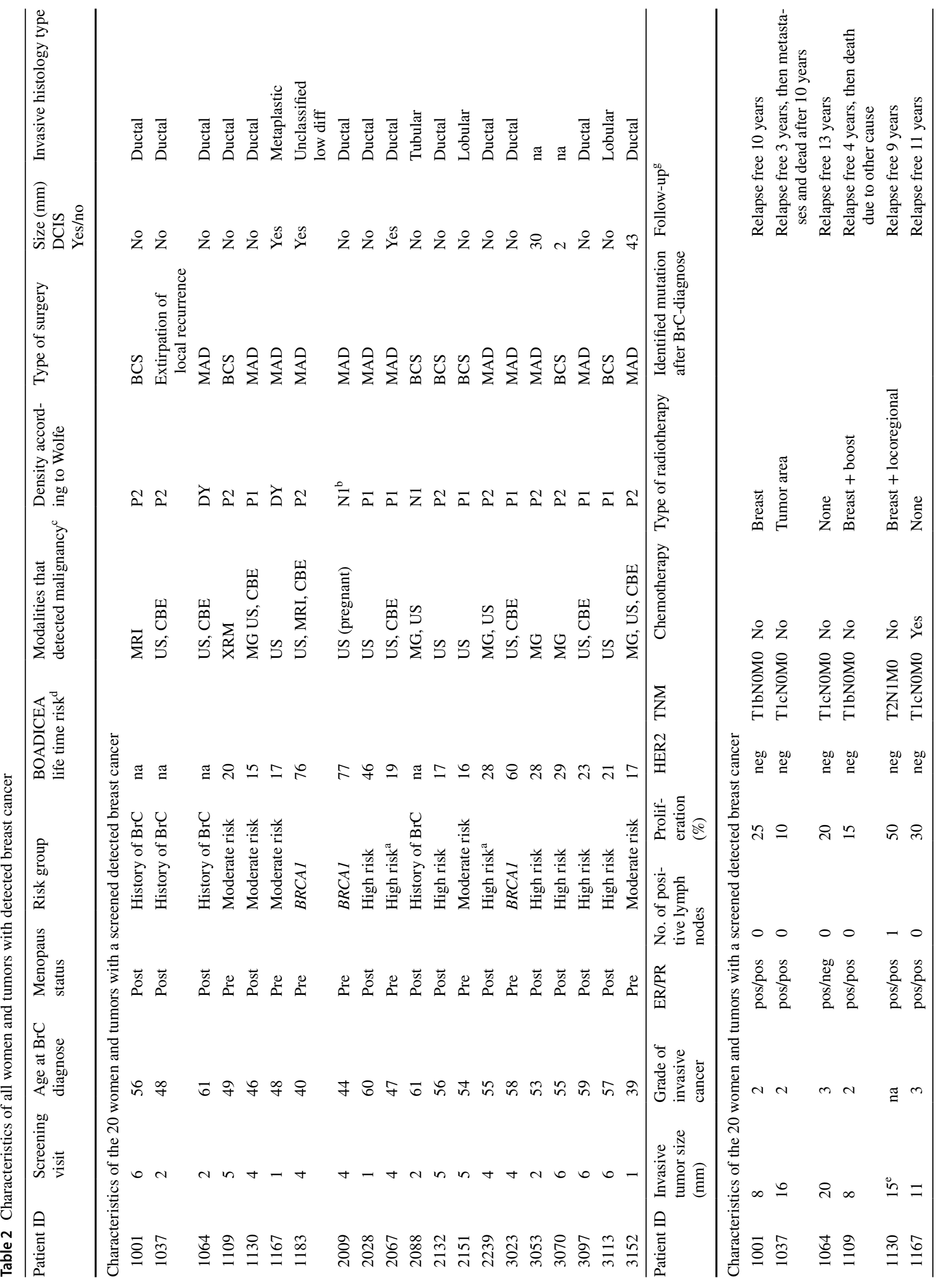




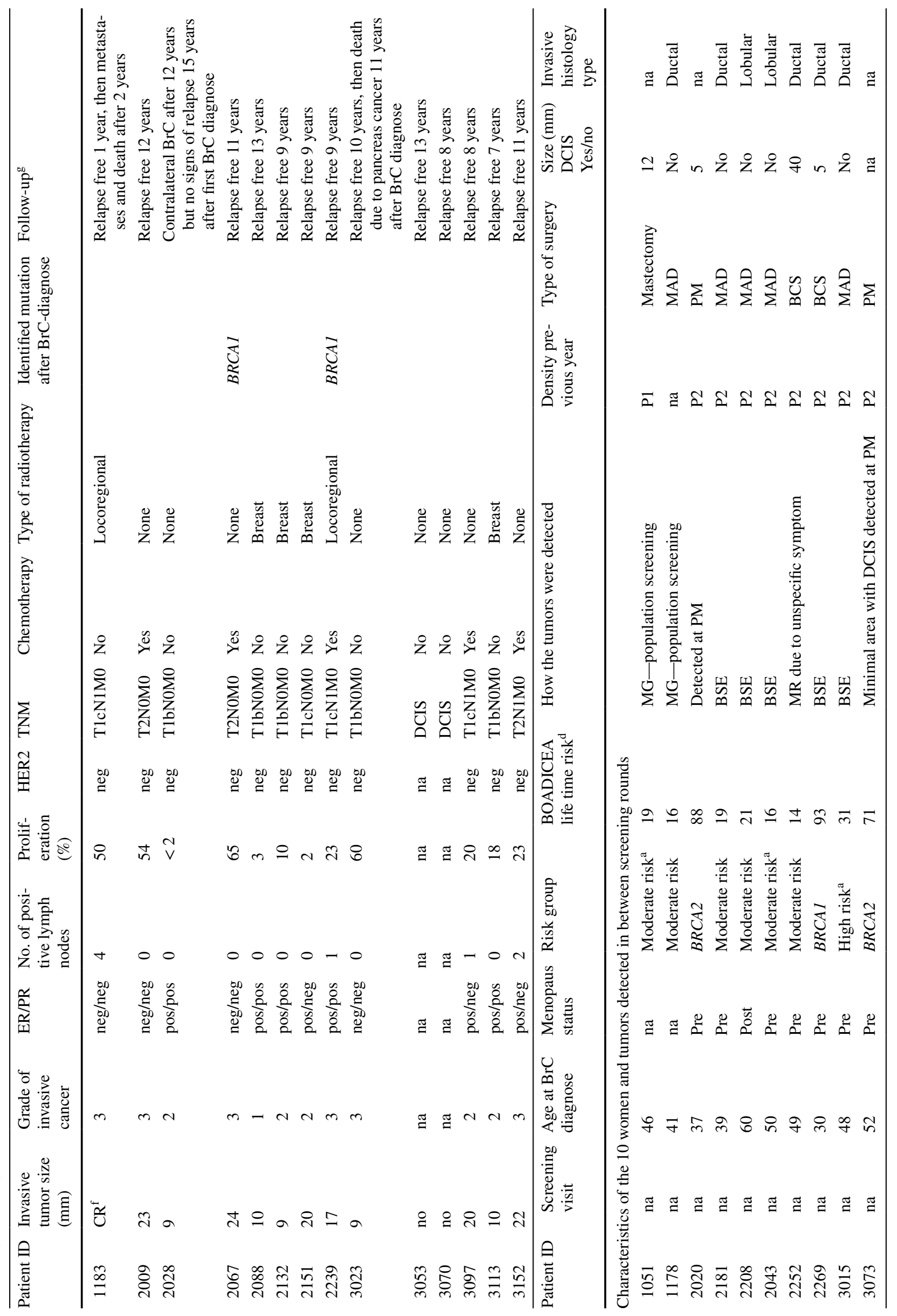




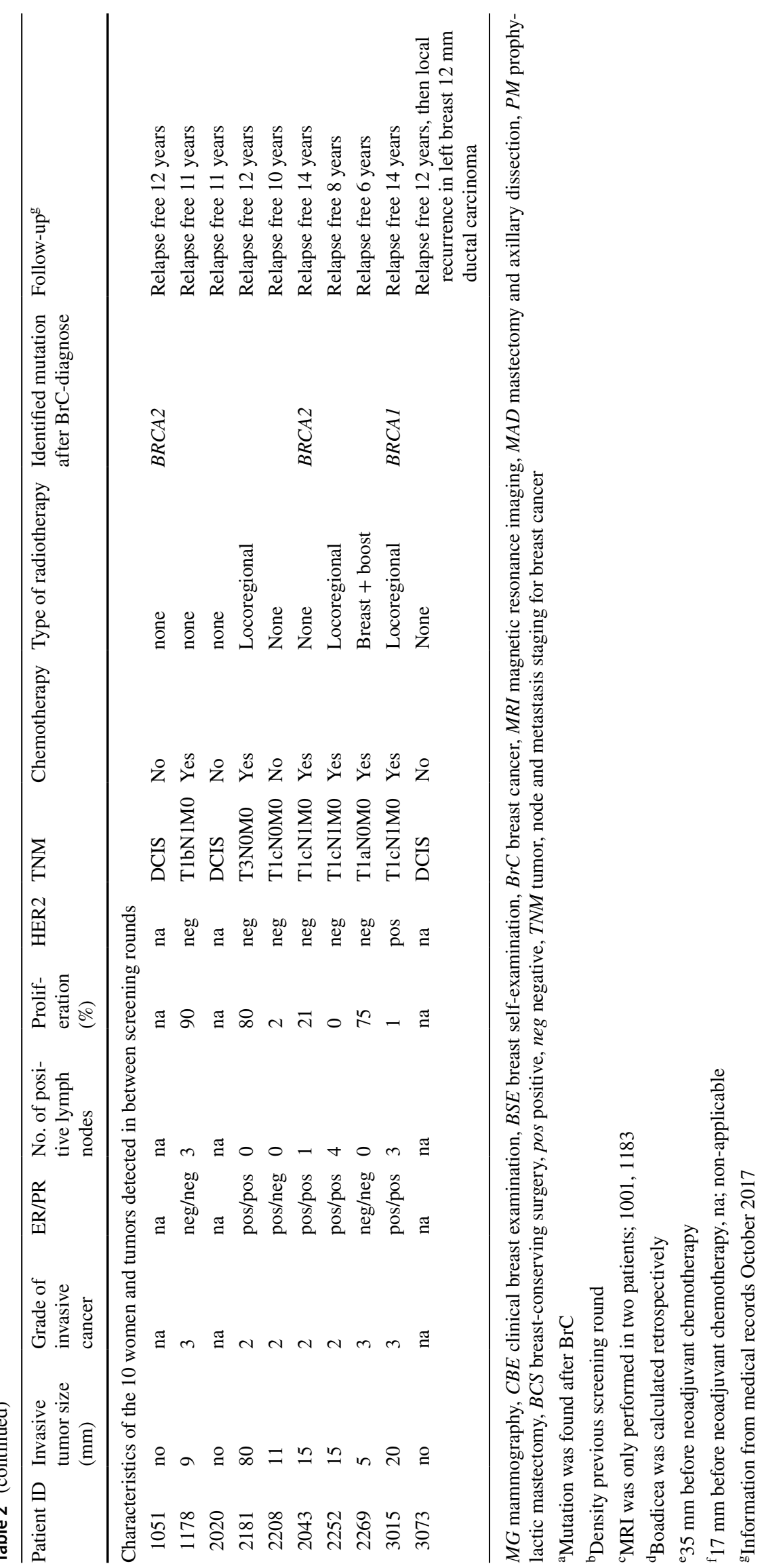



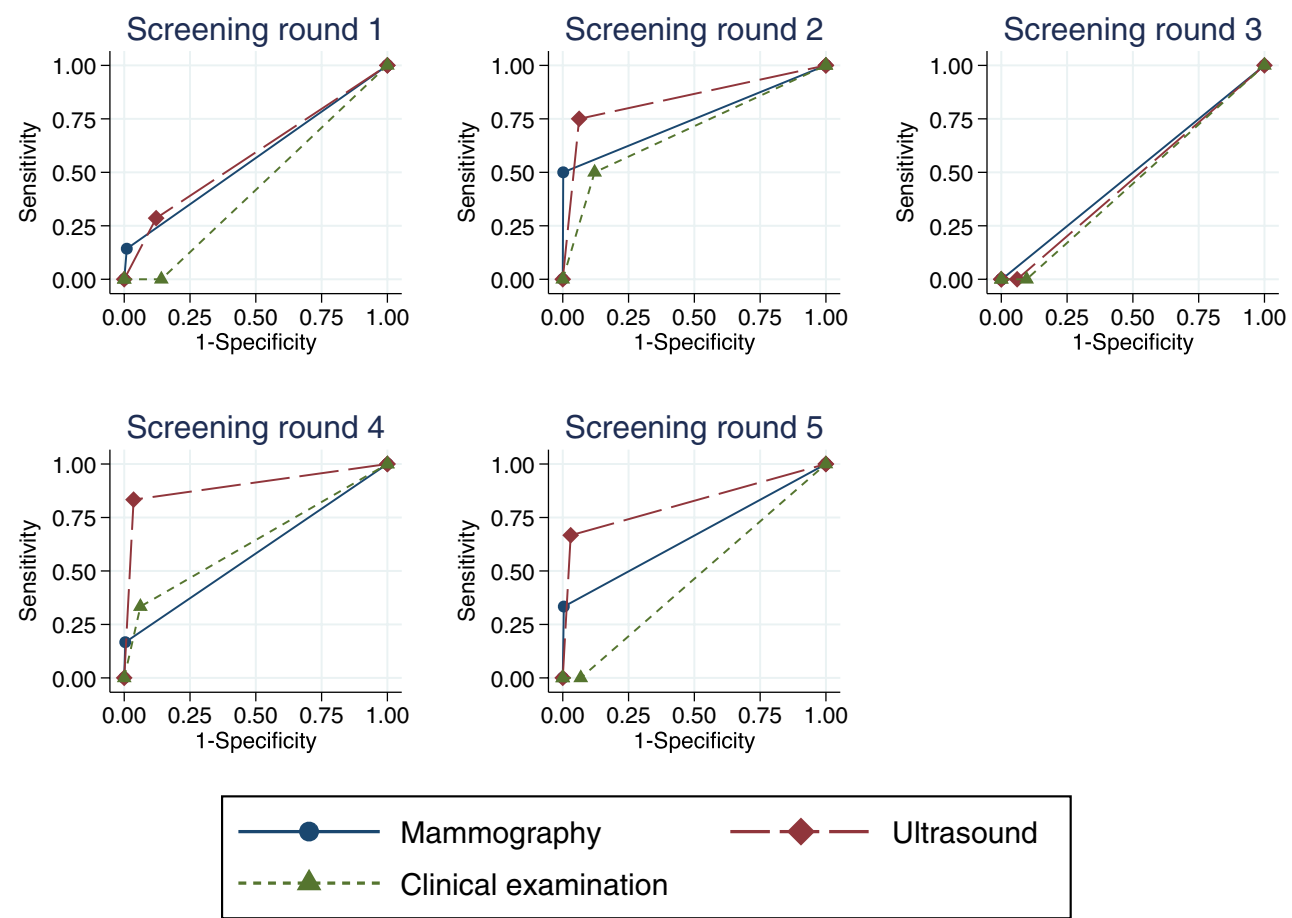

Fig. 2 Sensitivity and specificity of mammography, ultrasound, and clinical breast examination at each screening round

rounds. One explanation of this could be that despite the long experience of ultrasound imaging among the radiologists, the blinded procedure without having the mammograms available was novel for them, but after a few rounds their experience increased. However, only screening round 4 showed a significant difference in area under the curve between mammography and ultrasound imaging modalities. In addition, no consistent patterns in the receiver operating characteristics curves of the different screening modalities were observed across screening rounds. A possible limitation, of this study, was the low number of breast cancer cases. This low number prevented the use of elaborate statistical regression models to fully incorporate the longitudinal structure of the data (i.e., generalized mixed models) or subgroup-specific analyses stratified based on risk factors such as age or breast density. Therefore, we were not able to investigate the influence of breast density in the sensitivity analyses, although the correlation is well known $[8,14,23]$. Nevertheless, our results, in terms of sensitivity and specificity of mammography and ultrasound imaging, are similar to those reported by Kuhl et al. [11]. However, Kuhl et al. also included magnetic resonance imaging in their study and demonstrated a much higher sensitivity for magnetic resonance imaging than for mammography and ultrasound. Unfortunately, access to magnetic resonance imaging screening only became available for $B R C A 1$ or $B R C A 2$ carriers and women with a medical history of breast cancer half way through the study. Consequently, magnetic resonance imaging findings are not included in our sensitivity and specificity analyses.

The sensitivity and specificity of clinical breast examination were low in this study which is in accordance with the meta-analysis by Koster and Gotzsche [9]. Indeed, after the study was completed in 2012, clinical breast examination screening was consequently excluded in all types of surveillance program for women with increased hereditary risk of breast cancer in the Stockholm region (2 million inhabitants). In gene carriers, a yearly physician visit is optional for discussions about prophylactic surgery, psychological issues and clinical examination if desired by the patient. The positive consequences from this modification of the screening programs are of major importance since patients from all risk groups can avoid one check-up visit per year, and thus costs and time commitments. Medical providers could also benefit by avoiding these costs linked to out-patient visits. In addition, due to the high number of interval cancers (33\%) and tumors with lymph node metastases (36\%), and with $60 \%$ of the interval cancers detected in gene carriers, we conclude that the overall performance was poor. Therefore, it appears that the surveillance program for breast cancer mutation carriers should be modified to include screening with alternating magnetic resonance imaging and mammography and ultrasound at 6-month intervals. In 2013, updated international guidelines recommending annual magnetic resonance imaging for high risk individuals and mutation carriers [15] were also published. 
The decreasing number of referrals for cytology over time presented in Fig. 2 is explained by the novel working process for the ultrasound radiologists and the clinicians in that they were obliged to consider and code all breast lesions without support from any of the other imaging modalities. However, these different numbers over time was not a limitation for the sensitivity and specificity analyses though all code 2 or less were not included in these analyses. In contrast, the mammography radiologists had long experience of reading mammograms in a standalone procedure and hence, no difference was seen throughout the study in their referrals for cytology.

Many different methods of risk estimation [2] and risk categorization have been used in screening surveillance studies $[10,11,17]$. However, categorizing women into different groups relating to their hereditary risk of breast cancer is challenging. The risk estimation in this study was based on family history pedigree patterns. This distribution of the risk groups is similar to the distribution of risk groups in a study by Kriege et al. [10] who also used the modified Claus tables [6] for risk estimation. The risk estimation model Breast and Ovarian Analysis Disease Incidence and Carrier Estimation Algorithm [BOADICEA] has replaced the Claus tables in clinical practice, but use of the latter instead of the Claus tables for risk estimation would not have affected our findings significantly (all but one woman with breast cancer would have been included in our study if the BOADICEA risk model had been used).

Our findings indicate that a modified surveillance program may be used for individuals with increased hereditary risk for breast cancer. As neither mammography nor ultrasound performed yearly were sensitive enough to detect breast cancer consistently as a standalone modality in our study, we advise women with BOADICEA lifetime risk $<20 \%$ to adhere to the population screening program with biennial mammography. For women with $\geq 20$ to $29 \%$ lifetime risk according to BOADICEA a surveillance program, including annual mammography combined with ultrasound if indicated, may be sufficient. In high risk women with $\geq 30 \%$ lifetime risk in combination with high dense breasts, the same protocol as used in mutation carriers may be considered, namely screening every sixth months with magnetic resonance imaging at month 1 and mammography and ultrasound at month 6. Importantly, clinical breast examination may be omitted in all surveillance program for woman with hereditary increased risk of breast cancer, but could be optional for gene carriers.

Acknowledgements The authors wish to thank Kerstin Bjurling, Kerstin Moberg, Anders Adolfsson, Ulla Eriksson, Kersti Hjukström, Ulla Platten, Meta Rogestam, Margareta Ståhl, Louise Werelius, and Bo Nilsson for valuable assistance in this work. We also express our gratitude to all the women who participated.
Funding Financial support was provided through the Regional Agreement on Medical Training and Clinical Research (ALF) between the Stockholm County Council and Karolinska Institutet.

\section{Compliance with ethical standards}

Conflict of interest The authors declare that they have no conflict of interest.

Open Access This article is distributed under the terms of the Creative Commons Attribution 4.0 International License (http://creativecomm ons.org/licenses/by/4.0/), which permits unrestricted use, distribution, and reproduction in any medium, provided you give appropriate credit to the original author(s) and the source, provide a link to the Creative Commons license, and indicate if changes were made.

\section{References}

1. American College of Radiology (2003) Breast Imaging Reporting and Data System ${ }^{\circledR}$ (BI-RADS $\left.{ }^{\circledR}\right) 4$

2. Amir E, Freedman OC, Seruga B, Evans DG (2010) Assessing women at high risk of breast cancer: a review of risk assessment models. J Natl Cancer Inst 102:680-691. https://doi.org/10.1093 /jnci/djq088

3. Antoniou AC, Pharoah PP, Smith P, Easton DF (2004) The BOADICEA model of genetic susceptibility to breast and ovarian cancer. Br J Cancer 91:1580-1590. https://doi.org/10.1038/ sj.bjc. 6602175

4. Azavedo E, Svane G, Auer G (1989) Stereotactic fine-needle biopsy in 2594 mammographically detected non-palpable lesions. Lancet 1:1033-1036

5. Berg WA, Blume JD, Cormack JB, Mendelson EB, Lehrer D, Bohm-Velez M, Pisano ED, Jong RA, Evans WP, Morton MJ, Mahoney MC, Larsen LH, Barr RG, Farria DM, Marques HS, Boparai K, Investigators A (2008) Combined screening with ultrasound and mammography vs mammography alone in women at elevated risk of breast cancer. JAMA 299:2151-2163. https://doi. org/10.1001/jama.299.18.2151

6. Claus EB, Risch N, Thompson WD (1994) Autosomal dominant inheritance of early-onset breast cancer. Implications for risk prediction. Cancer 73:643-651

7. Eisinger F, Alby N, Bremond A, Dauplat J, Espie M, Janiaud P, Kuttenn F, Lebrun JP, Lefranc JP, Pierret J, Sobol H, StoppaLyonnet D, Thouvenin D, Tristant H, Feingold J (1998) Recommendations for medical management of hereditary breast and ovarian cancer: the French National Ad Hoc Committee. Ann Oncol 9:939-950

8. Kolb TM, Lichy J, Newhouse JH (2002) Comparison of the performance of screening mammography, physical examination, and breast US and evaluation of factors that influence them: an analysis of 27,825 patient evaluations. Radiology 225:165-175. https:// doi.org/10.1148/radiol.2251011667

9. Kosters JP, Gotzsche PC (2003) Regular self-examination or clinical examination for early detection of breast cancer. Cochrane Database Syst Rev. https://doi.org/10.1002/14651858.cd003373

10. Kriege M, Brekelmans CT, Boetes C, Besnard PE, Zonderland HM, Obdeijn IM, Manoliu RA, Kok T, Peterse H, TilanusLinthorst MM, Muller SH, Meijer S, Oosterwijk JC, Beex LV, Tollenaar RA, de Koning HJ, Rutgers EJ, Klijn JG, Magnetic Resonance Imaging Screening Study Group (2004) Efficacy of MRI and mammography for breast-cancer screening in women with a 
familial or genetic predisposition. N Engl J Med 351:427-437. https://doi.org/10.1056/nejmoa031759

11. Kuhl CK, Schrading S, Leutner CC, Morakkabati-Spitz N, Wardelmann E, Fimmers R, Kuhn W, Schild HH (2005) Mammography, breast ultrasound, and magnetic resonance imaging for surveillance of women at high familial risk for breast cancer. J Clin Oncol 23:8469-8476. https://doi.org/10.1200/JCO.2004 .00 .4960

12. Kuhl C, Weigel S, Schrading S, Arand B, Bieling H, Konig R, Tombach B, Leutner C, Rieber-Brambs A, Nordhoff D, Heindel W, Reiser M, Schild HH (2010) Prospective multicenter cohort study to refine management recommendations for women at elevated familial risk of breast cancer: the EVA trial. J Clin Oncol 28:1450-1457. https://doi.org/10.1200/JCO.2009.23.0839

13. Leach MO, Boggis CR, Dixon AK, Easton DF, Eeles RA, Evans DG, Gilbert FJ, Griebsch I, Hoff RJ, Kessar P, Lakhani SR, Moss SM, Nerurkar A, Padhani AR, Pointon LJ, Thompson D, Warren RM, MARIBS study group (2005) Screening with magnetic resonance imaging and mammography of a UK population at high familial risk of breast cancer: a prospective multicentre cohort study (MARIBS). Lancet 365:1769-1778. https://doi.org/10.1016 /S0140-6736(05)66481-1

14. Mandelson MT, Oestreicher N, Porter PL, White D, Finder CA, Taplin SH, White E (2000) Breast density as a predictor of mammographic detection: comparison of interval- and screen-detected cancers. J Natl Cancer Inst 92:1081-1087

15. National Collaborating Centre for Cancer (2013) Familial breast cancer: classification and care of people at risk of familial breast cancer and management of breast cancer and related risks in people with a family history of breast cancer. Update of clinical guideline 14 and 41. Clinical guideline 164. NCCC, London

16. Riedl CC, Ponhold L, Flory D, Weber M, Kroiss R, Wagner T, Fuchsjager M, Helbich TH (2007) Magnetic resonance imaging of the breast improves detection of invasive cancer, preinvasive cancer, and premalignant lesions during surveillance of women at high risk for breast cancer. Clin Cancer Res 13:6144-6152. http s://doi.org/10.1158/1078-0432.CCR-07-1270

17. Schmutzler RK, Rhiem K, Breuer P, Wardelmann E, Lehnert M, Coburger S, Wappenschmidt B (2006) Outcome of a structured surveillance programme in women with a familial predisposition for breast cancer. Eur J Cancer Prev 15:483-489. https://doi. org/10.1097/01.cej.0000220624.70234.14

18. StataCorp (2013) Stata Statistical Software: Release 13. StataCorp LP, College Station

19. Trop I, Lalonde L, Mayrand MH, David J, Larouche N, Provencher D (2010) Multimodality breast cancer screening in women with a familial or genetic predisposition. Curr Oncol 17:28-36

20. Warner E, Plewes DB, Hill KA, Causer PA, Zubovits JT, Jong RA, Cutrara MR, DeBoer G, Yaffe MJ, Messner SJ, Meschino WS, Piron CA, Narod SA (2004) Surveillance of BRCA1 and BRCA2 mutation carriers with magnetic resonance imaging, ultrasound, mammography, and clinical breast examination. JAMA 292:13171325. https://doi.org/10.1001/jama.292.11.1317

21. Weinstein SP, Localio AR, Conant EF, Rosen M, Thomas KM, Schnall MD (2009) Multimodality screening of high-risk women: a prospective cohort study. J Clin Oncol 27:6124-6128. https:// doi.org/10.1200/JCO.2009.24.4277

22. Wolfe JN (1976) Breast patterns as an index of risk for developing breast cancer. AJR Am J Roentgenol 126:1130-1137. https://doi. org/10.2214/ajr.126.6.1130

23. Wolfe JN (1976) Risk for breast cancer development determined by mammographic parenchymal pattern. Cancer 37:2486-2492 